\title{
Simulation of nonlinear benchmarks and sheet metal forming processes using linear and quadratic solid-shell elements combined with advanced anisotropic behavior models
}

\author{
Peng Wang ${ }^{1, a}$, Hocine Chalal ${ }^{1}$ and Farid Abed-Meraim ${ }^{1}$ \\ ${ }^{1}$ LEM3, UMR CNRS 7239 - Arts et Métiers ParisTech, 4, rue Augustin Fresnel, 57078 Metz Cedex 03, France
}

\begin{abstract}
A family of prismatic and hexahedral solid-shell (SHB) elements with their linear and quadratic versions is presented in this paper to model thin 3D structures. Based on reduced integration and special treatments to eliminate locking effects and to control spurious zero-energy modes, the SHB solid-shell elements are capable of modeling most thin 3D structural problems with only a single element layer, while describing accurately the various throughthickness phenomena. In this paper, the SHB elements are combined with fully 3D behavior models, including orthotropic elastic behavior for composite materials and anisotropic plastic behavior for metallic materials, which allows describing the strain/stress state in the thickness direction, in contrast to traditional shell elements. All SHB elements are implemented into ABAQUS using both standard/quasi-static and explicit/dynamic solvers. Several benchmark tests have been conducted, in order to first assess the performance of the SHB elements in quasi-static and dynamic analyses. Then, deep drawing of a hemispherical cup is performed to demonstrate the capabilities of the SHB elements in handling various types of nonlinearities (large displacements and rotations, anisotropic plasticity, and contact). Compared to classical ABAQUS solid and shell elements, the results given by the SHB elements show good agreement with the reference solutions.
\end{abstract}

\section{Introduction}

Nowadays, thin structures are increasingly used in engineering applications, and especially in automotive industries. For the design of new products while optimizing their forming processes with reasonable cost, the finite element simulation has become an indispensable practice. However, due to the large aspect ratio (length to thickness) of thin structures, the conventional solid and shell elements suffer from various locking phenomena both in linear and nonlinear analyses. In order to obtain accurate and reliable numerical results, much effort has been dedicated in recent decades to the development of efficient locking-free finite elements.

The recent concept of solid-shell elements attracted much attention due to their outstanding advantages compared to traditional solid and shell elements. They are based on a fully three-dimensional (3D) formulation with only displacements as degrees of freedom. Combined with the reduced-integration technique, various methods have been proposed in the literature to eliminate most locking phenomena [1-9], among which the assumedstrain method (ASM), the enhanced assumed strain (EAS) formulation, and the assumed natural strain (ANS) approach.

In this contribution, a family of assumed-strain based solid-shell elements (SHB elements) is briefly presented within a unified formulation. It consists of a linear eight-

\footnotetext{
a Corresponding author: peng.wang@ensam.eu
}

node hexahedral element (SHB8PS) and a linear six-node prismatic element (SHB6), and their quadratic counterparts (SHB20) and (SHB15), respectively. The SHB elements are combined with various types of constitutive equations, including classical isotropic elastic behavior, orthotropic elastic behavior for composite materials, and anisotropic plastic behavior for metallic materials. All SHB elements have been implemented into ABAQUS static/implicit and dynamic/explicit software packages in order to extend their applications to nonlinear quasi-static and dynamic analyses. A variety of nonlinear benchmark tests and a complex sheet metal forming process have been simulated to assess the performance of the proposed SHB elements.

\section{SHB solid-shell elements}

The proposed SHB elements are based on a fully threedimensional formulation using an in-plane reducedintegration scheme and the assumed-strain method. This SHB family is composed of a linear six-node prismatic element (SHB6) and an eight-node hexahedral element (SHB8PS), along with their quadratic counterparts (SHB15) and (SHB20), respectively [3, 5, 10-12]. Figure 1 shows the reference geometry of the four SHB solidshell elements as well as the location of their integration points. The latter are distributed along the local direction 
$\zeta$, which is designated as the thickness direction (see Figure 1). Note that the number of through-thickness integration points is chosen freely, depending on the problem complexity, which avoids using several element layers for an accurate description of the various throughthickness phenomena.

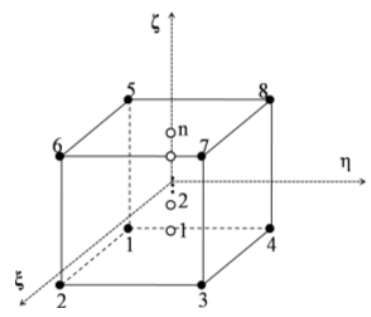

(a) SHB8PS

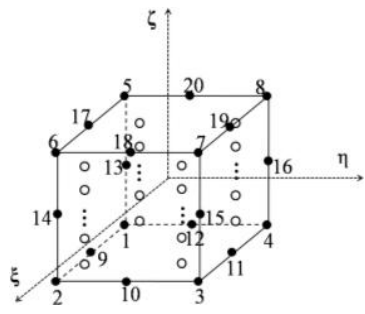

(c) SHB20

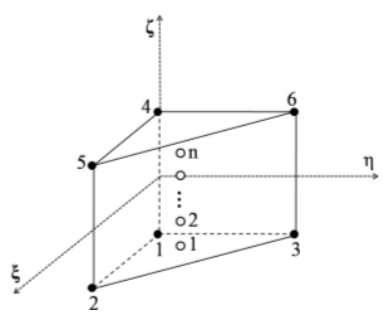

(b) SHB6

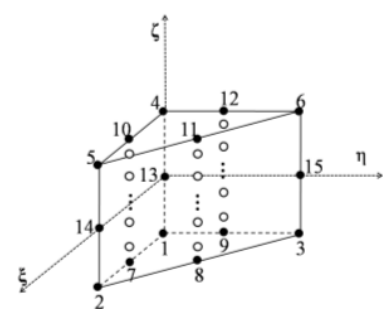

(d) SHB15
Figure 1. Reference geometry and location of integration points for the SHB elements.

\subsection{Finite element formulation}

The assumed-strain formulation of the SHB solid-shell elements is derived from the simplified form of the $\mathrm{Hu}-$ Washizu variational principle [13]. In terms of assumedstrain rate $\dot{\overline{\boldsymbol{\varepsilon}}}$, interpolated stress $\boldsymbol{\sigma}$, nodal velocities $\dot{\mathbf{d}}$, and external nodal forces $\mathbf{f}^{\text {ext }}$, this principle writes

$$
\pi(\dot{\bar{\varepsilon}})=\int_{\Omega_{e}} \delta \dot{\overline{\boldsymbol{\varepsilon}}}^{T} \cdot \boldsymbol{\sigma} d \Omega-\delta \dot{\mathbf{d}}^{T} \cdot \mathbf{f}^{e x t}=0 .
$$

By introducing the discretized gradient operator B , which contains the derivatives of the shape functions, the strain field $\boldsymbol{\varepsilon}$ can be related to the nodal displacement field $\mathbf{d}$ by the following expression:

$$
\boldsymbol{\varepsilon}=\mathbf{B} \cdot \mathbf{d} \text {. }
$$

In the case of linear SHB elements (i.e., SHB6 and SHB8PS), a $\overline{\mathbf{B}}$ matrix is introduced, which is obtained by projection of the original operator $\mathbf{B}$ onto an appropriate sub-space in order to eliminate most locking phenomena. Accordingly, the assumed-strain rate $\dot{\overline{\boldsymbol{\varepsilon}}}$ can be expressed in terms of the $\overline{\mathbf{B}}$ matrix as

$$
\dot{\overline{\mathbf{\varepsilon}}}=\overline{\mathbf{B}} \cdot \dot{\mathbf{d}} \text {. }
$$

Replacing the above assumed-strain rate in the expression of the variational principle (Eq. 1), the element stiffness matrix $\mathbf{K}_{e}$ and internal forces $\mathbf{f}^{\text {int }}$ are derived as

$$
\mathbf{K}_{e}=\int_{\Omega_{e}} \overline{\mathbf{B}}^{T} \cdot \mathbf{C}^{e p} \cdot \overline{\mathbf{B}} d \Omega+\mathbf{K}_{G E O M}
$$

$$
\mathbf{f}^{\text {int }}=\int_{\Omega_{e}} \overline{\mathbf{B}}^{T} \cdot \boldsymbol{\sigma} d \Omega,
$$

where $\mathbf{K}_{G E O M}$ is the geometric stiffness matrix, which originates from the nonlinear part of the strain tensor [5]. As to matrix $\mathbf{C}^{e p}$, it corresponds to the elastic-plastic tangent modulus.

In addition to the above formulation of SHB elements, a standard mass matrix is added to the dynamic version of each SHB element.

\subsection{Constitutive modeling}

The finite element implementation of the above SHB formulation requires introducing several local frames for the calculation of the stiffness matrix and internal forces associated with the constitutive law considered (see Figure 2). The "element frame" is used to define the thickness direction with respect to the global frame, while the "material frame" is specifically introduced to integrate the constitutive equations.

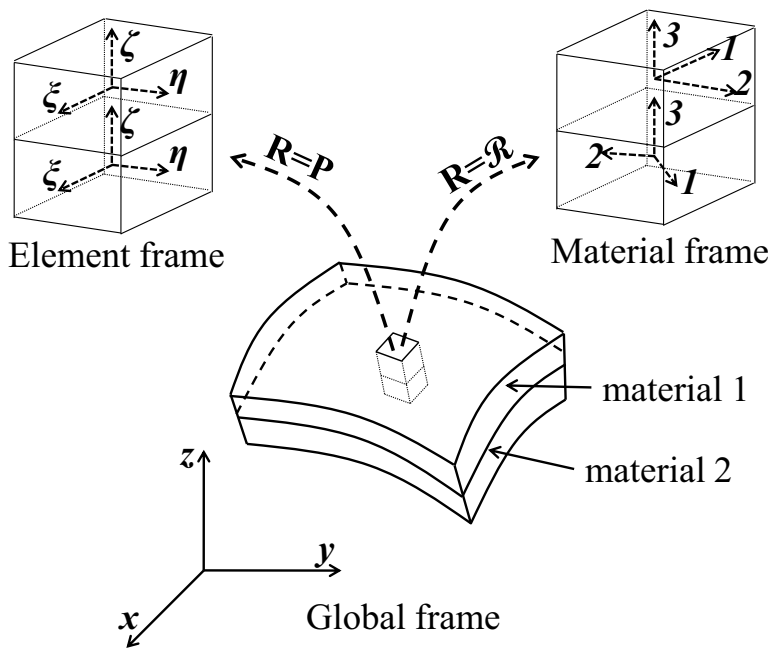

Figure 2. Illustration of the local frames used in the formulation of the SHB solid-shell elements.

\subsubsection{Elastic material behavior}

In this work, two typical 3D elastic behavior models are considered: an isotropic elastic behavior model for standard isotropic materials and an orthotropic elastic behavior model for laminated composites. The rate form of the stress-strain relationship can be expressed using the hypoelastic law defined in the local material frame by

$$
\dot{\boldsymbol{\sigma}}=\mathbf{C}: \mathbf{D}^{e}
$$

where $\mathbf{D}^{e}$ is the elastic strain rate tensor. Matrix $\mathbf{C}$ is the fourth-order elasticity tensor, which is defined in the element local frame. In the case of isotropic elastic behavior, a degenerated elasticity tensor $\mathbf{C}$ is used in the SHB formulation in order to approach plane-stress-type behavior. Its expression is given by 


$$
\mathbf{C}=\left[\begin{array}{cccccc}
\bar{\lambda}+2 \mu & \bar{\lambda} & 0 & 0 & 0 & 0 \\
\bar{\lambda} & \bar{\lambda}+2 \mu & 0 & 0 & 0 & 0 \\
0 & 0 & E & 0 & 0 & 0 \\
0 & 0 & 0 & \mu & 0 & 0 \\
0 & 0 & 0 & 0 & \mu & 0 \\
0 & 0 & 0 & 0 & 0 & \mu
\end{array}\right]
$$

with $\mu=\frac{E}{2(1+v)}, \quad \bar{\lambda}=\frac{E v}{1-v^{2}}$,

where $E$ is the Young modulus and $v$ is the Poisson ratio.

For laminated composite materials, a classical orthotropic elasticity law is adopted in the formulation of the SHB elements. Similar to the isotropic elasticity tensor, the orthotropic elasticity tensor $\overline{\mathbf{C}}$ is defined in the element local frame within the orthotropic axes as

$$
\overline{\mathbf{C}}^{-1}=\left[\begin{array}{cccccc}
\frac{1}{E_{1}} & -\frac{v_{21}}{E_{2}} & -\frac{v_{31}}{E_{3}} & 0 & 0 & 0 \\
-\frac{v_{12}}{E_{1}} & \frac{1}{E_{2}} & -\frac{v_{32}}{E_{3}} & 0 & 0 & 0 \\
-\frac{v_{13}}{E_{1}} & -\frac{v_{23}}{E_{2}} & \frac{1}{E_{3}} & 0 & 0 & 0 \\
0 & 0 & 0 & \frac{1}{G_{12}} & 0 & 0 \\
0 & 0 & 0 & 0 & \frac{1}{G_{13}} & 0 \\
0 & 0 & 0 & 0 & 0 & \frac{1}{G_{23}}
\end{array}\right],
$$

with $v_{i j} E_{j}=v_{j i} E_{i}$ for $i, j=1,2,3$,

where $E_{i}$ represents the Young modulus in the $i$ thdirection, $v_{i j}$ is the Poisson ratio associated with the $i$ th and $j$ th-directions, and $G_{12}, G_{13}$ and $G_{23}$ are the shear moduli.

Considering an initial rotation angle $\theta$ of the orthotropic axes with respect to the global frame, the projected elasticity tensor can be obtained using the following expressions:

$$
\hat{\mathbf{C}}=\mathbf{T}(\theta) \cdot \overline{\mathbf{C}} \cdot \mathbf{T}(\theta)^{T},
$$

where the rotation matrix $\mathbf{T}(\theta)$ has the following form:

$$
\mathbf{T}(\theta)=\left[\begin{array}{cccccc}
c^{2} & s^{2} & 0 & 2 c s & 0 & 0 \\
s^{2} & c^{2} & 0 & -2 c s & 0 & 0 \\
0 & 0 & 1 & 0 & 0 & 0 \\
-c s & c s & 0 & c^{2}-s^{2} & 0 & 0 \\
0 & 0 & 0 & 0 & c & -s \\
0 & 0 & 0 & 0 & s & -s
\end{array}\right],
$$

with $c=\cos (\theta)$ and $s=\sin (\theta)$.

\subsubsection{Anisotropic elastic-plastic behavior}

When plastic behavior is considered for the material, the strain rate tensor $\mathbf{D}$ is partitioned into an elastic part $\mathbf{D}^{\mathrm{e}}$ and a plastic part $\mathbf{D}^{\mathrm{p}}$. The latter is defined using the classical plastic flow rule

$$
\mathbf{D}^{\mathrm{p}}=\dot{\lambda} \frac{\partial f}{\partial \boldsymbol{\sigma}},
$$

where $f$ represents the plastic yield surface, and $\dot{\lambda}$ is the plastic multiplier whose expression is determined using the consistency condition.

In this work, anisotropic plastic behavior is taken into account using the Hill'48 quadratic yield surface. The yield condition is written in the following form:

$$
f=\bar{\sigma}_{\text {eq }}-Y \leq 0,
$$

where $\bar{\sigma}_{\text {eq }}=\sqrt{\left(\boldsymbol{\sigma}^{\prime}-\boldsymbol{\alpha}\right): \mathbf{H}:\left(\boldsymbol{\sigma}^{\prime}-\boldsymbol{\alpha}\right)}$ is the equivalent stress, in which $\sigma^{\prime}$ represents the deviatoric part of the Cauchy stress, and $\mathbf{H}$ is the fourth-order tensor that contains the six anisotropy coefficients of Hill'48 criterion. Isotropic and kinematic hardening is taken into account using the internal variables $Y$ and $\boldsymbol{\alpha}$, respectively.

\section{Numerical examples and results}

In this section, several popular benchmark problems and a complex sheet metal forming process are simulated to evaluate the performance of the SHB elements in situations involving large rotations, large strain, material nonlinearity, and double-sided contact. In the case of elastic benchmark problems, only two integration points through the thickness are used in the simulations, while five integration points are considered in the case of elasto-plastic problems. The results obtained with the SHB elements are consistently compared with reference solutions as well as with the numerical results yielded by ABAQUS solid and shell elements.

In the following benchmark tests, the nomenclature used for the mesh is: $\left(\mathrm{N}_{1} \times \mathrm{N}_{2}\right) \times \mathrm{N}_{3}$ for hexahedral elements, where $\mathrm{N}_{1}$ is the number of elements along the length, $\mathrm{N}_{2}$ is the number of elements along the width and $\mathrm{N}_{3}$ is the number of elements along the thickness direction. For the prismatic elements, however, the nomenclature used is $\left(\mathrm{N}_{1} \times \mathrm{N}_{2} \times 2\right) \times \mathrm{N}_{3}$, due to the in-plane sub-division of a rectangular element into two triangles. Similarly, the nomenclature used for triangular ABAQUS shell elements is $\mathrm{N}_{1} \times \mathrm{N}_{2} \times 2$, while the nomenclature for quadrilateral ABAQUS shell elements is $\mathrm{N}_{1} \times \mathrm{N}_{2}$.

\subsection{Quasi-static benchmark problems}

\subsubsection{Simply supported elastic square plate}

Figure 3 illustrates a simply supported isotropic elastic square plate subjected to a central concentrated force. The detailed information about the geometric dimensions and the elastic material parameters is given in Figure 3. 
The maximum of the concentrated load $\mathrm{F}$ is equal to 300 . Due to symmetry, only one quarter of the plate is analyzed.

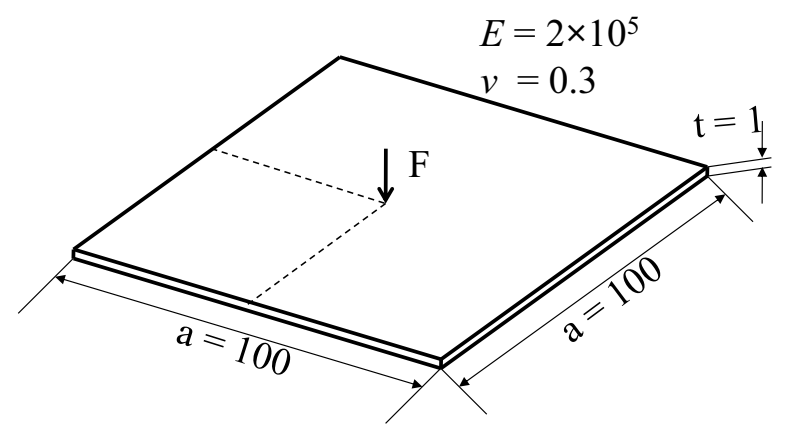

Figure 3. Geometry and elastic material parameters for the simply supported square plate subjected to a concentrated force.

The load-displacement results at the central point of the plate given by the SHB elements are compared in Figure 4 with the ABAQUS elements and also with the reference solutions taken from $[14,15]$.

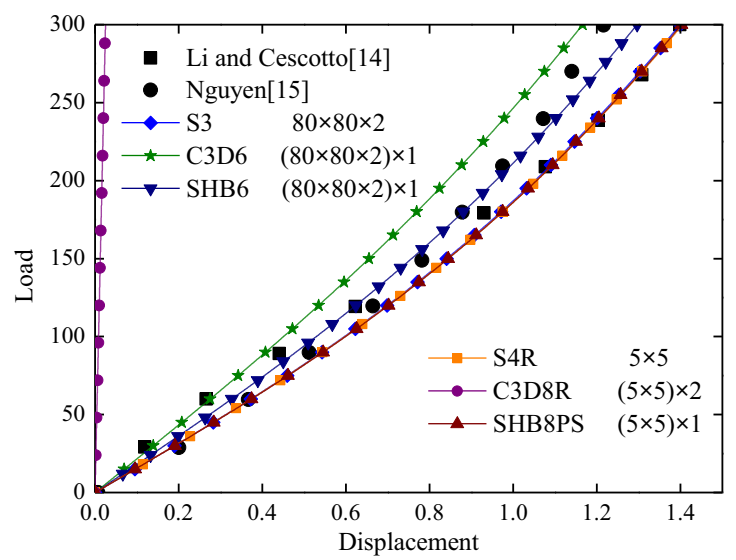

(a) Linear elements

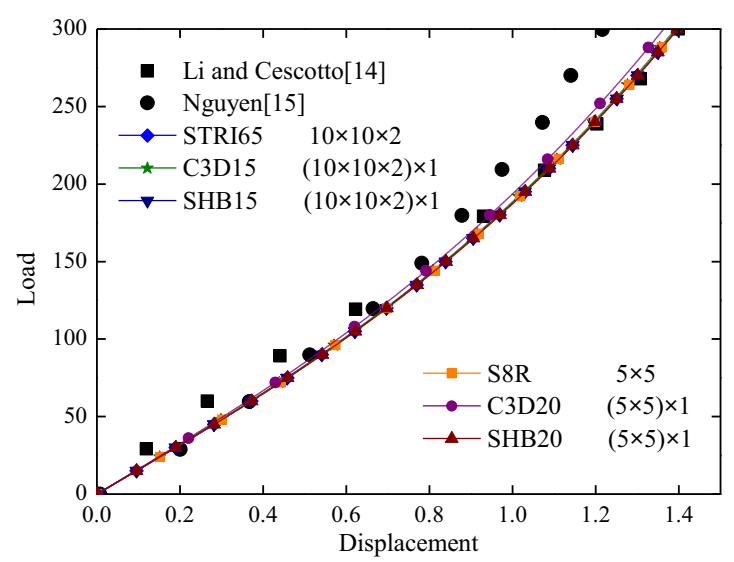

(b) Quadratic elements

Figure 4. Load-displacement curves for the simply supported square plate.

In the case of triangular-based linear elements (Figure 4a), the results given by the SHB6 solid-shell element are closer to the reference solutions than those given by the ABAQUS prismatic solid element (C3D6), while the results yielded by the ABAQUS triangular shell element (S3) are in agreement with one of the reference solutions (i.e., that taken from [14]). For the quadrangular-based linear elements, the load-displacement results given by the SHB8PS element are very close to those obtained with the ABAQUS quadrilateral shell element (S4R) as well as to the reference solution taken from [14]. Note that, using the same in-plane mesh as SHB8PS and S4R elements, the load-displacement curve given by the ABAQUS hexahedral solid element (C3D8R) lies far from the reference solution, which suggest resorting to much finer meshes for this element. In the case of quadratic elements (Figure $4 \mathrm{~b}$ ), the results given by the solid-shell elements SHB15 and SHB20 agree very well with the reference solution taken from [14], as well as with the results obtained with the ABAQUS quadratic elements (namely, the prismatic solid element C3D15, the triangular shell element STRI65, and the quadrilateral shell element S8R), while the hexahedral solid element C3D20 is slightly stiffer.

\subsubsection{Pinched elastic-plastic hemispherical shell}

A pinched hemispherical shell is considered here, which is loaded by alternating radial forces as shown in Figure 5. This test is very popular since it involves both geometric and material nonlinearities. Owing to the problem symmetry, only one quarter of the structure is modeled. In addition to the nomenclature defined previously, three partitions for the quarter of the hemispherical shell are created in order to achieve a relatively regular mesh (see Figure 5). This leads to the following nomenclature for this test: $\left(3 \times\left(\mathrm{N}_{1} \times \mathrm{N}_{2}\right)\right) \times \mathrm{N}_{3}$ for hexahedral elements, $\left(3 \times\left(\mathrm{N}_{1} \times \mathrm{N}_{2} \times 2\right)\right) \times \mathrm{N}_{3}$ for prismatic elements, $3 \times\left(\mathrm{N}_{1} \times \mathrm{N}_{2}\right)$ for quadrilateral shell elements, and $3 \times\left(\mathrm{N}_{1} \times \mathrm{N}_{2} \times 2\right)$ for triangular shell elements.

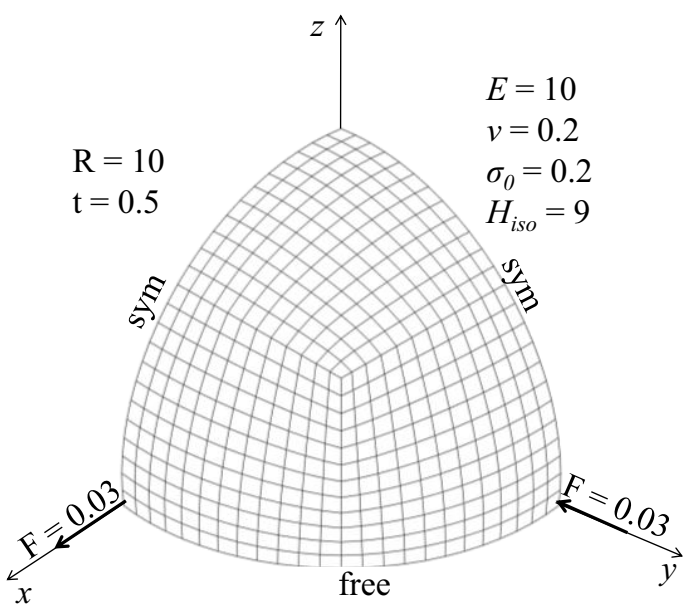

Figure 5. Geometry and material parameters for the pinched hemispherical shell.

In Figure 6, the displacements at the loading points in the $x$ and $y$ directions (namely, $\mathrm{U}$ and $\mathrm{V}$, respectively) obtained with the SHB elements are compared with those given by ABAQUS elements as well as with the reference solution taken from [16]. Overall, all SHB and ABAQUS elements provide accurate results with respect to the reference solution, except for the SHB6 and C3D6 elements, which exhibit some locking and which require finer meshes for this test problem. Note also that the proposed solid-shell elements are more efficient than 
their counterparts Abaqus solid elements, since the latter require several layers of elements to provide comparable accuracy (see Figure 6).

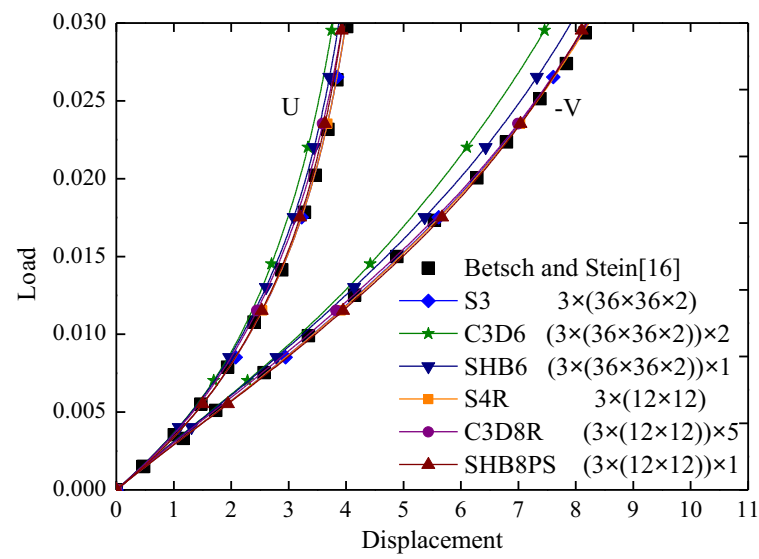

(a) Linear elements

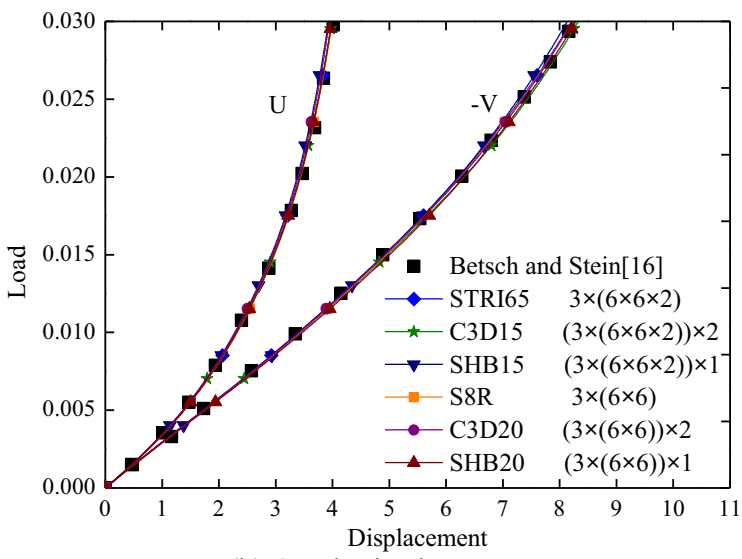

(b) Quadratic elements

Figure 6. Load-displacement curves for the pinched hemispherical shell.

\subsubsection{Clamped composite plate}

A laminated composite plate with orthotropic elastic behavior (see Subsection 2.2.1) is considered here. The plate is fully clamped at one end and subjected to a bending force at the free end. All geometric dimensions as well as orthotropic elastic parameters are given in Figure 7. For comparison purposes, two configurations of laminated composites, which correspond to two different stacking sequences $\left(0^{\circ} / 90^{\circ} / 0^{\circ}\right.$ and $\left.90^{\circ} / 0^{\circ} / 90^{\circ}\right)$, are considered. The load-deflection curves obtained with the SHB elements are compared in Figure 8 with those given by the ABAQUS elements as well as with the reference solutions taken from [17]. Note that the laminated composites are modeled with three layers in the case of prismatic and hexahedral elements due to the different stacking sequences through the thickness. The obtained results clearly show the excellent accuracy of the SHB elements when compared to ABAQUS elements and the reference solutions. This reveals the good performance of the proposed solid-shell elements when orthotropic behavior for laminated composites is considered.

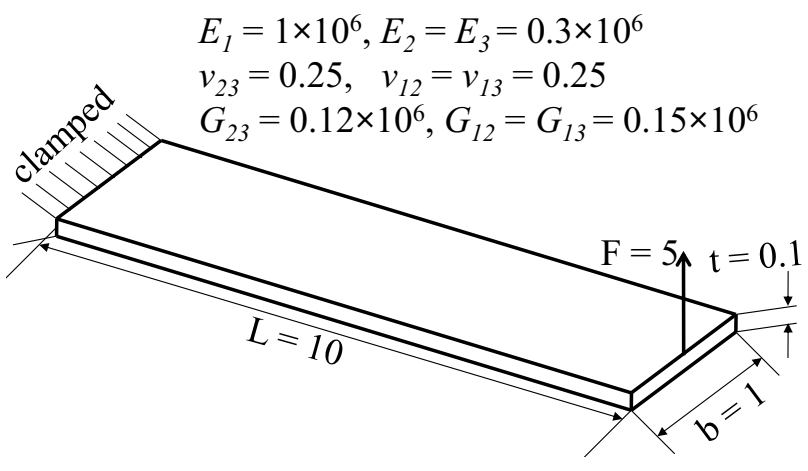

Figure 7. Orthotropic elastic parameters and geometric dimensions for the laminated composite cantilever plate.

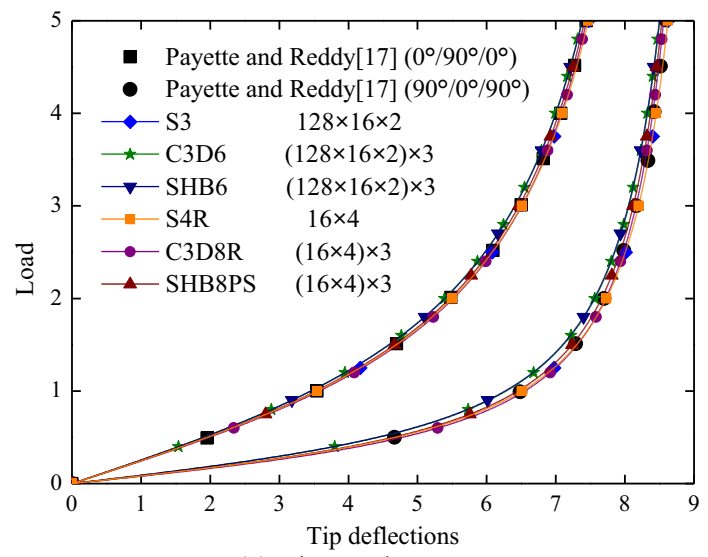

(a) Linear elements

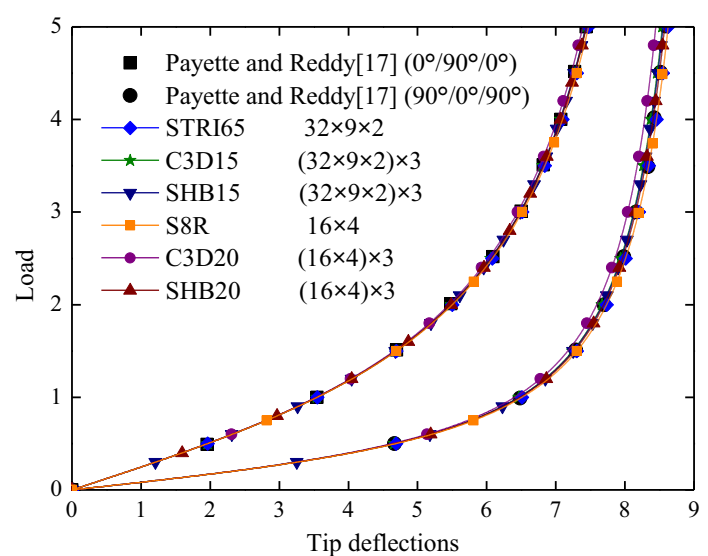

(b) Quadratic elements

Figure 8. Load-displacement curves for the laminated composite cantilever plate.

\subsection{Dynamic benchmark tests}

\subsubsection{Clamped elastic spherical cap}

The performance of the SHB elements is evaluated here within the framework of explicit dynamic analysis. The first explicit/dynamic problem is illustrated in Figure 9, which consists of a fully clamped spherical cap loaded by a constant concentrated force at its apex. Owing to the symmetry, only one quarter of the cap is discretized. The dynamic response in terms of central vertical displacement is analyzed in Figure 10 using the dynamic versions of the proposed SHB elements as well as 
ABAQUS explicit elements, which are compared to the reference solution taken from [18]. Note that, since no quadratic elements are available in ABAQUS/Explicit software package, the results given by the quadratic SHB elements are compared only with the reference solution (see Figure 10b). The results reveal that both the peak and the period of the response are well predicted using the proposed SHB elements as well as ABAQUS linear shell elements, while the solution yielded by the ABAQUS linear solid elements is found far from the reference solution during the second half-period.

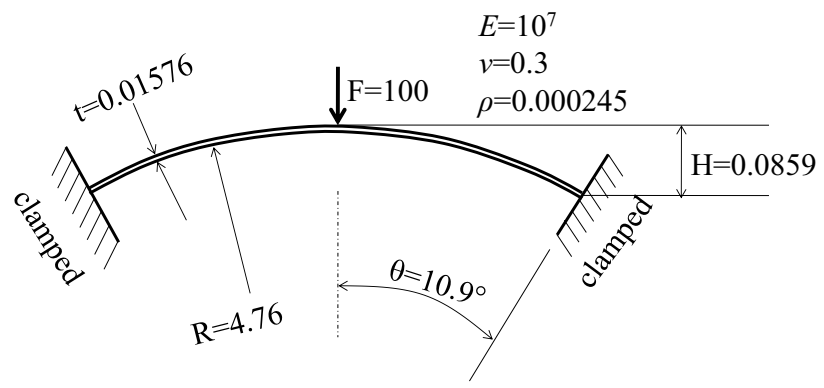

Figure 9. Elastic material parameters and geometric dimensions for the clamped spherical cap.

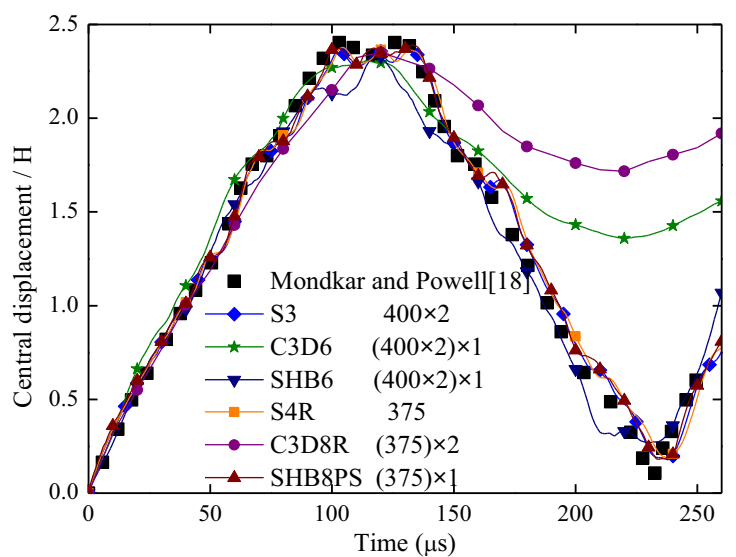

(a) Linear elements

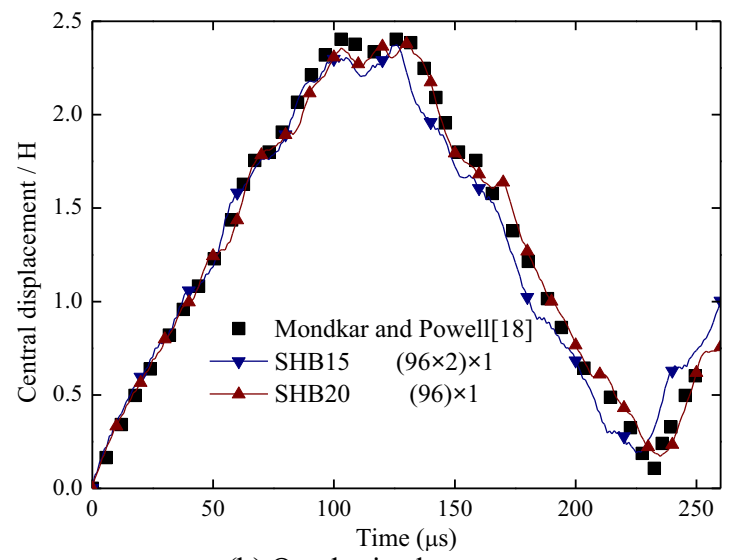

(b) Quadratic elements

Figure 10. Normalized displacement history for the point located at the apex of the clamped spherical cap.

\subsubsection{Simply supported composite plate}

The second dynamic benchmark test considered in this paper is a simply supported laminated plate, as shown in
Figure 11, which is composed of two layers $\left(0^{\circ} / 90^{\circ}\right)$. A concentrated force is applied at the middle of the plate. Figure 12 presents the dynamic responses in terms of central deflection of the plate obtained using the SHB elements and ABAQUS linear elements. Once again, excellent agreement with the reference solution taken from [19] is observed with the dynamic versions of the proposed SHB elements. The ABAQUS linear elements show good performance as well, except for the prismatic element C3D6, which requires finer meshes to provide accurate solution.

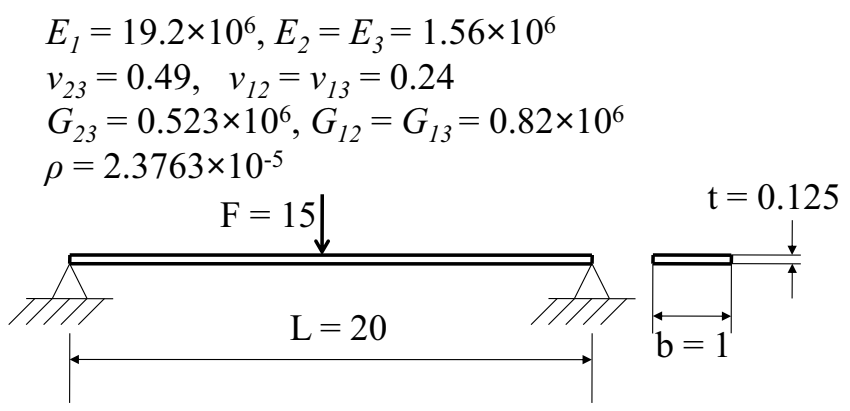

Figure 11. Geometry and material parameters for the simply supported laminated plate.

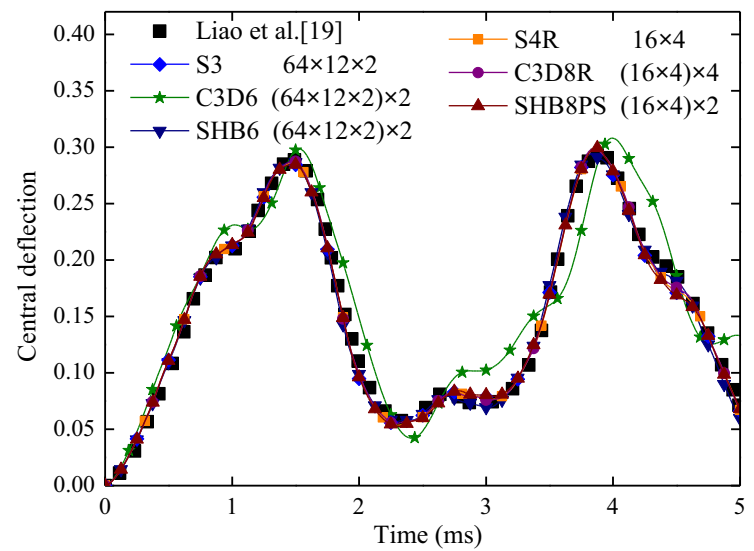

(a) Linear elements

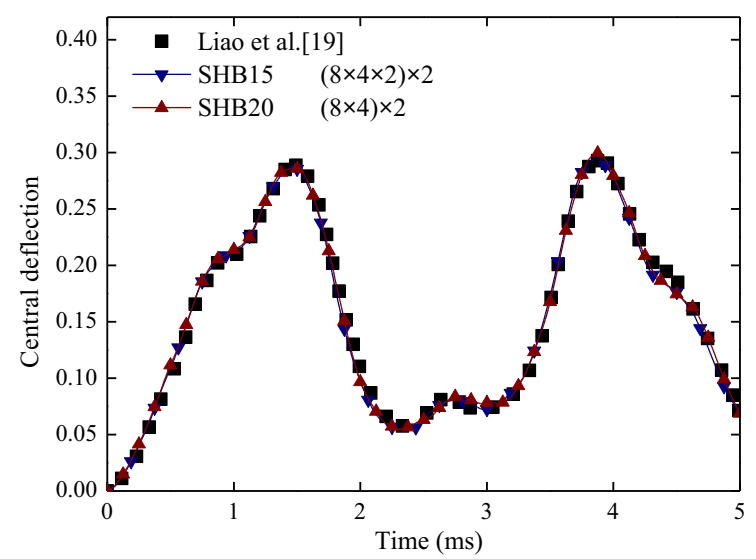

(b) Quadratic elements

Figure 12. Dynamic response curves for the simply supported laminated plate. 


\subsection{Sheet metal forming process}

This subsection is devoted to the simulation of deep drawing of a hemispherical cup in order to evaluate the capabilities of the SHB elements in modeling sheet metal forming processes. The forming setup and the dimensions of the tools are presented in Figure 13. A circular steel sheet with initial blank radius $\mathrm{R}=126.4 \mathrm{~mm}$ and thickness $\mathrm{t}=0.7 \mathrm{~mm}$ is considered in the simulations. The elastic material properties of the sheet are given by Young's modulus $E=210 \mathrm{GPa}$ and Poisson's ratio $v=0.3$. The stress-strain curve is described by the following Swift isotropic hardening law $\sigma=545\left(0.003+\varepsilon^{\mathrm{p}}\right)^{0.209}$, while kinematic hardening is not taken into account (see [20]). The Hill'48 plastic yield surface is considered here to model the plastic anisotropy of the studied material (see subsection 2.2.2). The associated coefficients are $\mathrm{F}=0.287$, $\mathrm{G}=0.357, \mathrm{H}=0.643, \mathrm{~L}=\mathrm{M}=1.5$, and $\mathrm{N}=1.306$. Since no blank-holder is used in this test, the blank is clamped all around its circumferential surface during the forming process. The friction coefficient between the tools and the blank is set to 0.15 . Owing to the axial symmetry, only one quarter of the circular sheet is modeled. The final deformed shape of the hemispherical cup using the SHB elements is shown in Figure 14.

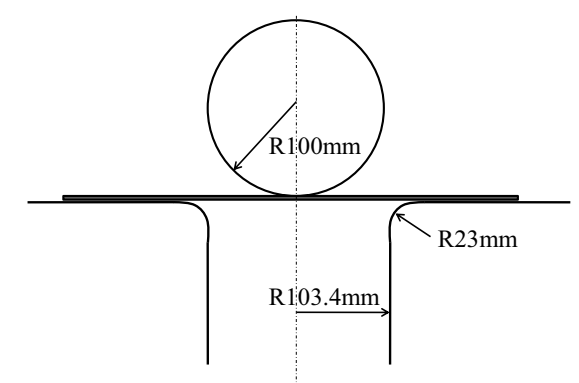

Figure 13. Hemispherical deep drawing setup.

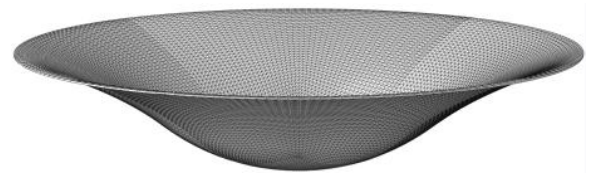

(a) SHB6

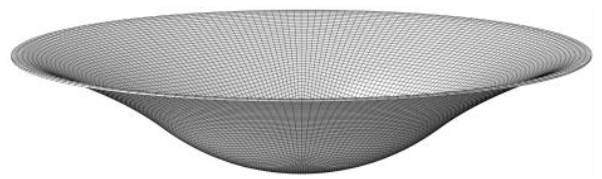

(b) SHB8PS

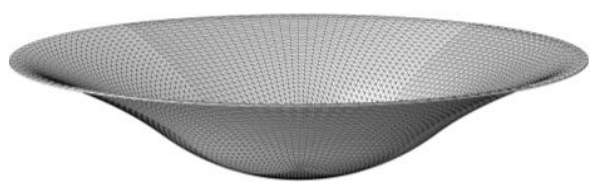

(c) SHB15

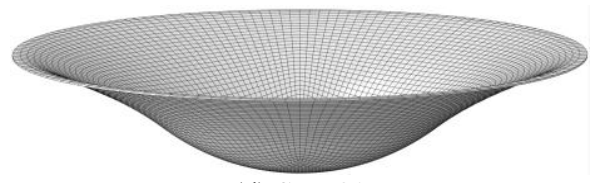

(d) SHB20

Figure 14. Final deformed shape for the hemispherical cup.
The profile of the thickness strain of the blank along the rolling direction and the reaction force of the punch are investigated in Figures 15 and 16, respectively, using the SHB solid-shell elements and only ABAQUS solid elements, and compared to the experimental results provided by Laurent [20]. Indeed, traditional shell elements do not allow outputting thickness strain variations due to their two-dimensional formulation.

From the predictions of the thickness strain profile in Figure 15, the SHB elements provide the closest results to the experimental one in terms of overall evolution and order of magnitude, while the results obtained with the ABAQUS solid elements are inaccurate, especially in the zone located about $40 \mathrm{~mm}$ from the centre of the blank. These contrasted results reveal that ABAQUS solid elements are not able to correctly handle the severe contact conditions between the blank and the punch in this zone, which lead to highly distorted meshes, as illustrated in Figure 16 with the ABAQUS quadratic element C3D20.

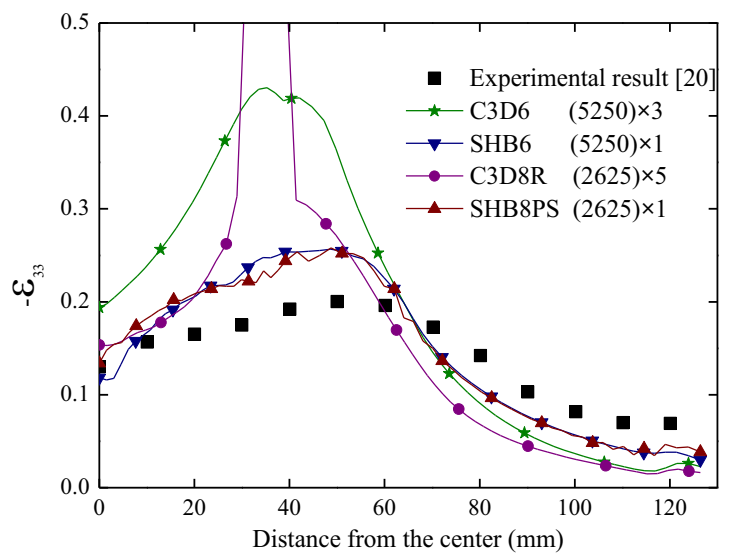

(a) Linear elements

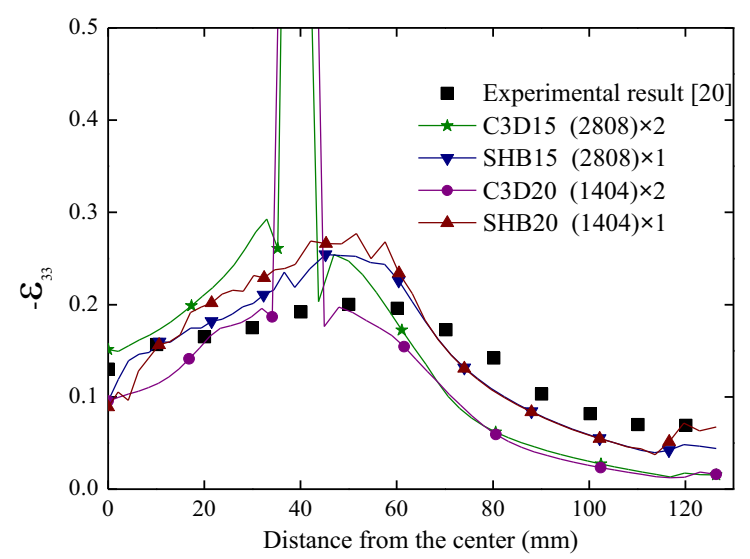

(b) Quadratic elements

Figure 15. Simulation results for the thickness strain.

Figure 17 shows the punch force, as predicted by all solid and solid-shell elements, together with the experimental results. Compared to ABAQUS elements, the results obtained with the SHB elements are in much better agreement with the experiments, which confirms once again the ability of the proposed solid-shell elements to model complex sheet metal forming processes using only a single layer of elements. 


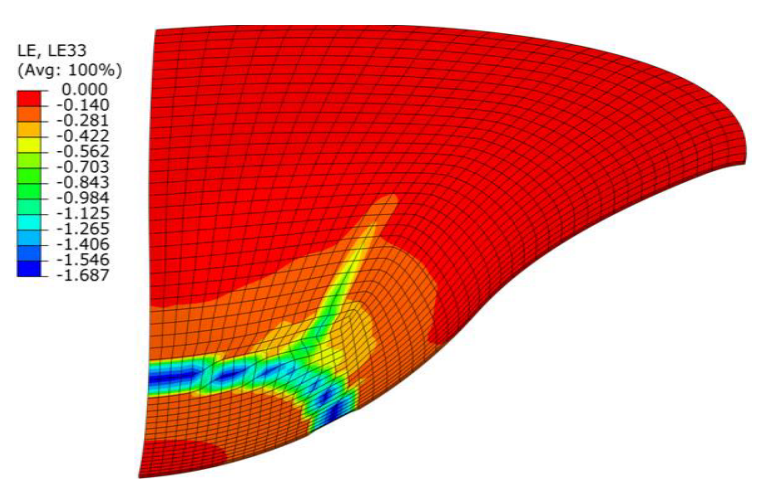

Figure 16. Final deformed cup using C3D20 element.

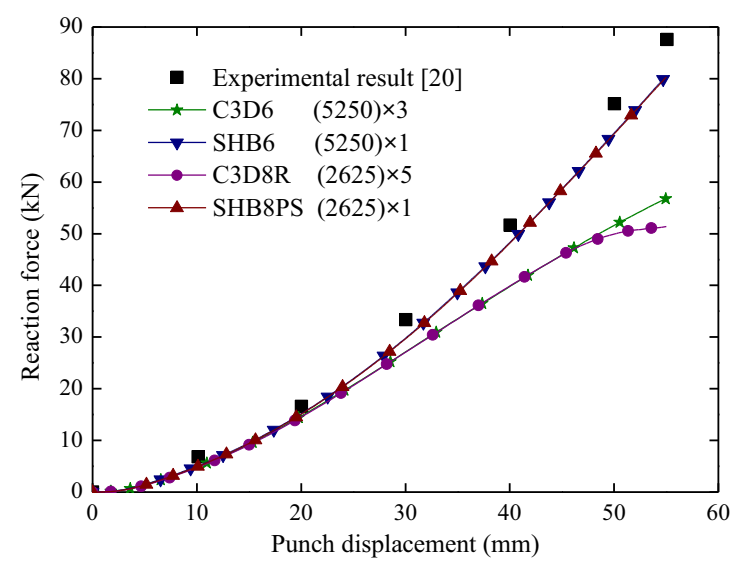

(a) Linear elements

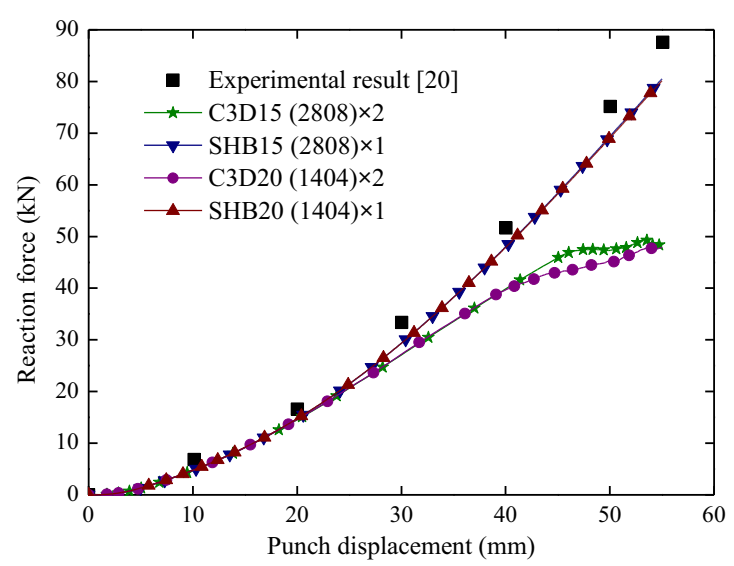

(b) Quadratic elements

Figure 17. Punch force evolution during the deep drawing of the hemispherical cup.

\section{Conclusions}

In this paper, the formulation of a family of assumedstrain-based solid-shell elements has been described, which has been then evaluated through quasi-static and dynamic analyses as well as simulation of challenging sheet metal forming processes.

In the formulation of the SHB elements, several local frames have been defined and used in their numerical implementation. Taking advantage of such modularity, various constitutive models have been coupled with these SHB elements, including isotropic behavior, orthotropic elastic behavior for laminated composite materials, and anisotropic plastic behavior for metallic materials.
A series of selective and representative benchmark tests, involving different types of materials, loading and boundary conditions, has been conducted to assess the performance of the SHB elements in quasi-static and dynamics analyses. Also, complex deep drawing of a hemispherical cup has been considered to evaluate the capabilities of the proposed SHB elements in handling various types of nonlinearities (geometric, material, and due to contact). Adopting equivalent in-plane meshes and numbers of integration points in the thickness direction, the SHB elements performed much better than their ABAQUS counterparts, in both types of analyses (quasistatic and dynamic). Moreover, the variety of numerical results presented in this paper reveal the good capabilities of the SHB elements and their wide prospective applications in the simulation of thin 3D structures.

\section{References}

1. C. Cho, H.C. Park, S.W. Lee, Finite Elem. Anal. Des. 29, 121-135 (1998)

2. R. Hauptmann, K. Schweizerhof, Int. J. Numer. Meth. Engng. 42, 49-70 (1998)

3. F. Abed-Meraim, A. Combescure, Comput. \& Struct. 80, 791-803 (2002)

4. R.P.R Cardoso, J.W. Yoon, M. Mahardika, S. Choudhry, R.J. Alves de Sousa, R.A. Fontes Valente, Int. J. Numer. Meth. Engng. 75, 156-187 (2008)

5. F. Abed-Meraim, A. Combescure, Int. J. Numer. Meth. Engng. 80, 1640-1686 (2009)

6. M. Schwarze, I.N. Vladimirov, S. Reese, Comput. Methods Appl. Mech. Engng. 200, 454-476 (2011)

7. F.G. Flores, Comput. Methods Appl. Mech. Engng. 266, 81-97 (2013)

8. M. Pagani, S. Reese, U. Perego, Comput. Methods Appl. Mech. Engng. 268, 141-159 (2014)

9. Q. Xie, K.Y. Sze, Y.X Zhou, Finite Elem. Anal. Des. 106, 85-102 (2015)

10. V.D. Trinh, F. Abed-Meraim, A. Combescure, J. Mech. Sci. Technol. 25, 2345-2364 (2011)

11. A. Salahouelhadj, F. Abed-Meraim, H. Chalal, T. Balan, Arch. Appl. Mech. 82, 1269-1290 (2012)

12. F. Abed-Meraim, V.D. Trinh, A. Combescure, Computing 95, 373-394 (2013)

13. J.C. Simo, T.J.R. Hughes. J. Appl. Mech. 53, 51-54 (1986)

14. K.P. Li, J.L. Cescotto, Comput. Methods Appl. Mech. Engng. 141, 157-204 (1997)

15. N.H. Nguyen, Ph.D. Thesis, Université de Liège, Belgium (2009)

16. P. Betsch, E. Stein, Comput. Mehods Appl. Mech. Engng. 179, 215-245 (1999)

17. G.S. Payette, J.N. Reddy, Comput. Methods Appl. Mech. Engng. 278, 664-704 (2014)

18. D.P. Mondkar, G.H. Powell, Int. J. Numer. Methods Engng. 11, 499-520 (1977)

19. C.L. Liao, J.N. Reddy, S.P. Engelstad, Int. J. Numer. Methods Engng. 26, 1843-1854 (1988)

20. H. Laurent. Ph.D. Thesis, Université du Maine, France (1996) 